\title{
FLORÍSTICA DE UM FRAGMENTO VEGETACIONAL DA ÁREA DE PROTEÇÃO AMBIENTAL DO BURITI DO MEIO, CAXIAS, MARANHÃO
}

Antônio Edmilson Camelo Júnior ${ }^{1}$, Guilherme Sousa da Silva², Gonçalo Mendes da Conceição $^{3}$

1. Acadêmico do curso de Ciências Biológicas Licenciatura, da Universidade Estadual do Maranhão - Maranhão/Brasil (antonioedmilsom@hotmail.com)

2. Mestrando de Pós-Graduação em Botânica do Instituto Nacional de Pesquisas da Amazônia - INPA, Manaus-AM, Brasil

3. Professor Dr. do Centro de Estudos Superiores de Caxias/CESC, da Universidade Estadual do Maranhão/UEMA, Maranhão/Brasil; Programa de Pós-Graduação em Biodiversidade, Ambiente e Saúde/PPGBAS

Recebido em: 15/04/2017 - Aprovado em: 22/07/2017 - Publicado em: 31/07/2017 DOI: 10.18677/Agrarian_Academy_2017a26

\begin{abstract}
O trabalho tem por finalidade caracterizar a Flora de um fragmento de vegetação da Área de Proteção Ambiental Municipal do Buriti do Meio, Caxias, Maranhão. A APA Municipal do Buriti do Meio possui uma área de 58.347,30 ha, sendo criado pela Lei n 1.540/2004 de 25 de março de 2004. O método florístico foi realizado através de levantamentos compostos por três fases: o trabalho de campo, com a realização de excursões à APA no período de outubro de 2015 à junho 2016 para coleta do material botânico; a análise e herborização do material botânico e por fim a identificação através de chaves taxonômicas e confirmação por especialistas. Foram obtidas 9 espécies, 78 gêneros e 32 famílias botânicas, a família mais representativa foi a família Fabaceae com 29 espécies, seguida por Malvaceae com sete espécies. Com o levantamento florístico elencou-se uma nova ocorrência para o Estado do Maranhão, espécie esta pertencente à família Fabaceae, Aeschynomene indica L. Quanto aos espécimes, a espécie mais representativa foi Pseudobombax marginatum (St. Hill) Rob., com 20 espécimes elencadas. Assim observa-se que a área vegetacional é bem diversa, e que o hábito de crescimento árvore é predominante. A APA por estar sob constate pressão antrópica é sujeita à drásticas mudanças, e medidas de conservação e de proteção devem ser tomadas, com a finalidade de proteger o patrimônio vegetal ali presente.
\end{abstract}

RESUMO

PALAVRAS-CHAVE: Cerrado, Fabaceae, Vegetação.

\section{FLORISTICS OF A FRAGMENT OF VEGETATION OF ENVIRONMENTAL PROTECTION AREA FROM BURITI DO MEIO, CAXIAS, MARANHÃO}

\begin{abstract}
The purpose of this work is to characterize the Flora of a fragment of vegetation of the Municipal Environmental Protection Area of Buriti do Meio, Caxias, Maranhão. The municipal enviromental protection area of Buriti do Meio has an area of $58.347,30$ ha, being created by law $n^{\circ} 1.540 / 2004$ of march 25 of 2004. The floristic method was realized through surveys composed of three phases: the field work, with
\end{abstract}


the realization of excursions to the Environmental Protection Area in the period from October 2015 to June 2016 to collect the botanical material; the analysis and herborization of the botanical material and finally the identification through taxonomic keys and confirmation by specialists. Were obtained 89 species, 78 genera and 32 botanical families, the most representative family was the Fabaceae family with 29 species, followed by Malvaceae with seven species. With the floristic survey was listed a new occurrence for the State of Maranhão, a species belonging to the family Fabaceae, Aeschynomene indica L. As for the specimens, the most representative species was Pseudobombax marginatum (St. Hill) Rob., with 20 specimens listed. Thus it is observed that the vegetation area is quite diverse, and that the habit of tree growth is predominant. The Environmental Protection Area for being under constant anthropic pressure is subject to drastic changes, measures of conservation and protection must be taken, with the purpose of protecting the plant patrimony present there.

KEYWORDS: Savannah, Fabaceae, Vegetation.

\section{INTRODUÇÃO}

A vegetação é o elemento mais evidente de uma paisagem, conferindo a fisionomia típica aos diferentes domínios fitogeográficos terrestres e oferecendo suporte à sobrevivência da fauna. Os primeiros estudos sobre vegetação se valiam em relatos escritos, desenhos e listagem de espécies para caracterizar as diferentes vegetações e biomas do mundo e eram essencialmente qualitativos (FELFILI et al., 2013).

Por sua vez, a vegetação brasileira, pela pujança e pela versatilidade de suas formas, pela riqueza florística e, ainda, pelos expressivos, marcantes e diversificados quadros de paisagens, representando modelos fisionômicos peculiares, continuamente chamou a atenção de estudiosos das mais diversas áreas. Estudos voltados à esfera da vegetação brasileira possibilitaram a elaboração de sistemas fitogeográficos, que buscaram descrever o perfil vegetacional e da riqueza florística, na qual possibilitou o estabelecimento dos relevantes padrões geomorfológicos, compartimentando o território brasileiro em seus domínios morfoclimáticos (FERNANDES, 2003).

Entender a florística e a organização comunitária da vegetação natural têm um grande valor significativo, tanto para o avanço de modelos de conservação e manejo de áreas remanescentes como para o restabelecimento de áreas perturbadas ou degradadas (SALIS et al., 1994). Ao lado de seu grande potencial de aplicação, levantamentos da composição florística e da estrutura comunitária da vegetação natural são de fundamental importância para o desenvolvimento da teoria ecológica e fitogeográfica, pois, além de gerarem informações cruciais sobre a distribuição geográfica das espécies, permitem que se amplie o conhecimento sobre a abundância das espécies em diferentes locais, fornecendo bases consistentes para a criação de unidades de conservação (GOMES et al., 2004).

Dentre os domínios fitogeográficos brasileiros o Cerrado localiza-se principalmente no Planalto Central do Brasil, ocupando $24 \%$ do território nacional, com aproximadamente dois milhões de quilômetros quadrados. O domínio é a segunda maior formação vegetal brasileira depois da Amazônia, sendo a savana tropical mais rica do mundo em biodiversidade concentrando um terço da biodiversidade nacional e $5 \%$ da flora e da fauna mundial, com cerca de 10 mil espécies vegetais (SANTOS et al., 2010).

AGRARIAN ACADEMY, Centro Científico Conhecer - Goiânia, v.4, n.7; p.269 2017 
A cobertura vegetal Maranhense reflete uma particular influência das condições de transição climática, entre o clima amazônico e o semi-árido nordestino, sendo considerada um ecótono (CONCEIÇÃO \& CASTRO, 2009). O Estado do Maranhão abrange cinco Mesorregiões Geográficas, sendo uma região significativa em termo de biodiversidade, por apresentar formações vegetacionais de Cerrado, Florestas Estacionais e Florestas ombrófilas, garantindo um berço de espécies botânicas, ainda pouco estudadas (MONTES , 1997).

O conhecimento sobre a distribuição e organização da biodiversidade, nas comunidades do Cerrado, é ainda reduzido. Estas informações são de grande importância para avaliar os impactos antrópicos, planejar a criação de unidades de conservação e a adoção de técnicas de manejo (FELFILI \& SILVA-JÚNIOR, 2001). Com a perspectiva de aumentar o nível de conhecimento sobre a vegetação do Maranhão, esse trabalho objetivou estudar e caracterizar a composição Florística, do componente arbóreo-arbustivo da Área de Proteção Ambiental Municipal do Buriti do Meio, Caxias, Maranhão.

\section{MATERIAL E MÉTODOS}

\section{Localização e Caracterização da Área de Estudo}

A Área de Proteção Ambiental Municipal do Buriti do Meio foi criada pela Lei no 1.540/2004 de 25 de março de 2004, localizada no Projeto de Assentamento do Buriti do Meio e Santa Rosa, no $2^{\circ}$ Distrito, afastada a $35 \mathrm{~km}$ do perímetro urbano da cidade de Caxias, presente nas seguintes coordenadas 04⒌'48,1"S e $43^{\circ} 06^{\prime} 49,2$ 'W, com extensão territorial de $58.347,30$ ha (Figura. 1). A estrutura vegetacional do espaço de estudo é constituída por diferentes fisionomias de cerrado natural e antropizado, com solos arenosos, ácidos, pobres em nutrientes e frágeis (Figura. 2) (IBGE, 2010).

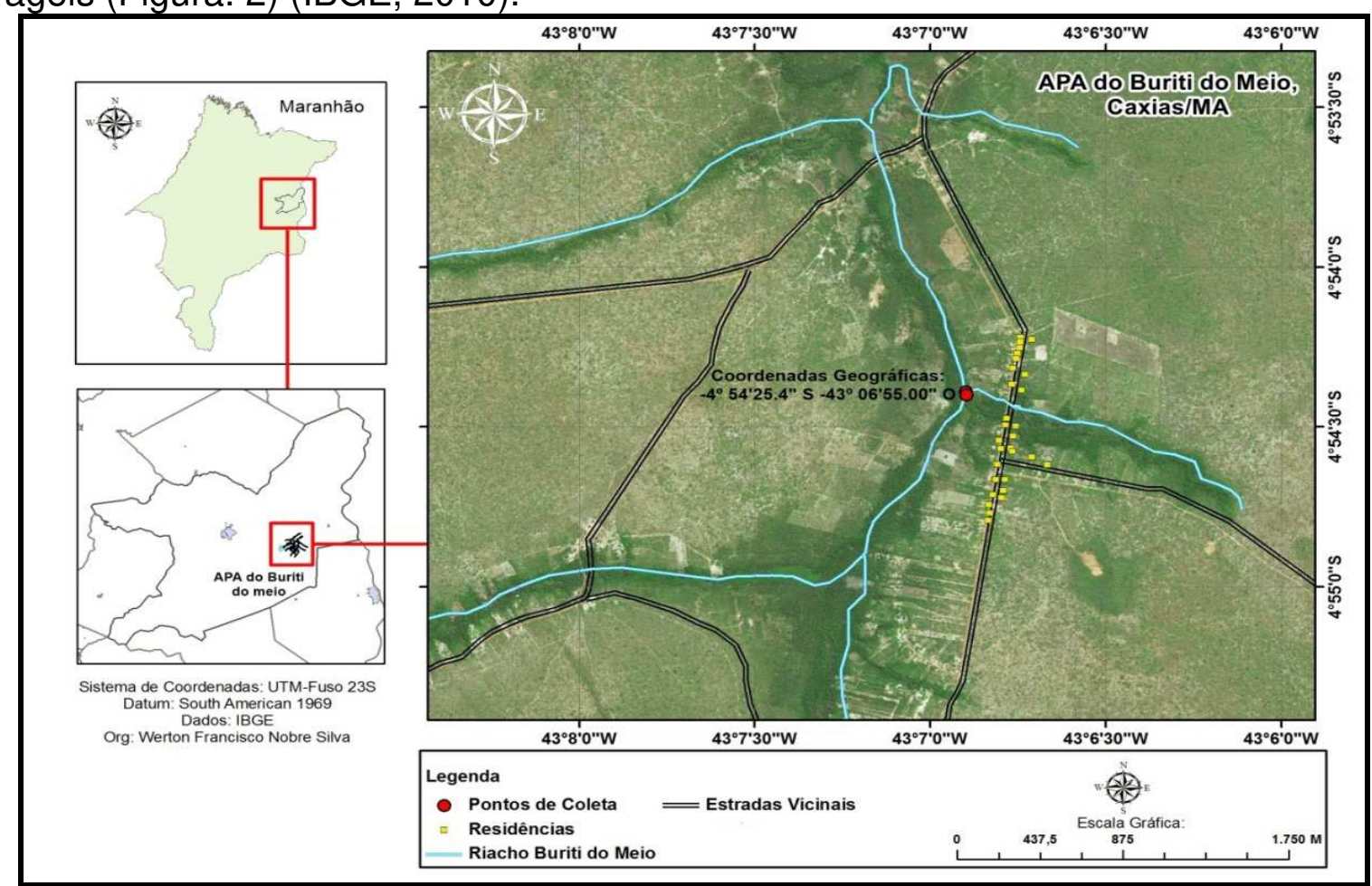

FIGURA 1. Mapa da Área de Proteção Ambiental do Buriti do Meio. Fonte: IBGE, 2006; Organização: SILVA (2016). 


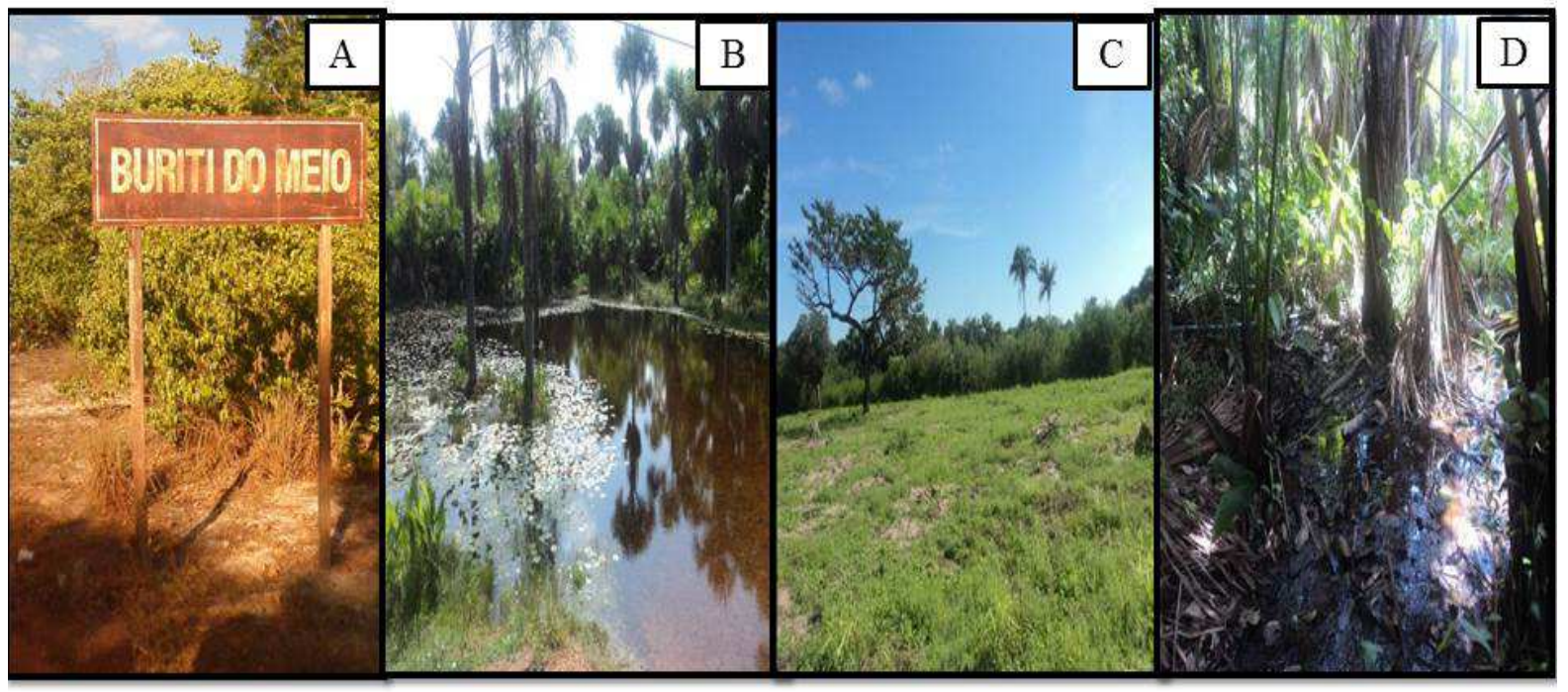

FIGURA 2. Características do Povoado Buriti do Meio. A) Placa com localização do Povoado; B) Riacho do Povoado; C) Área de Campo Limpo; D) Área de Mata de Galeria. Fonte: SILVA, (2016).

\section{MÉTODO FLORÍSTICO}

O levantamento florístico foi composto principalmente por três fases: o trabalho de campo, a análise e identificação, e a preparação do material botânico. Foram executadas expedições nos meses de Outubro de 2015 à Junho 2016 na APA, estabelecendo um total de nove coletas destinadas à obtenção de dados em campo. Os espécimes coletados estavam em estado fértil, possuindo flores ou fruto. Dessa forma, as saídas a campo contemplaram vários meses do ano para que assim, o maior número possível de espécies seja encontrado no estágio reprodutivo (com flores ou frutos) para facilitar a sua identificação. As etapas ocorreram das seguintes formas:

\section{Trabalho de campo}

Com o auxílio de tesoura de poda foram coletados os espécimes em estado fértil, coletando no mínimo cinco ramos de cada indivíduo (Fig. 3-A), que foram acondicionados em sacos plásticos e etiquetados de acordo com a sequência de coleta. Para cada espécime coletado foi realizada uma serie de anotações como: data e local de coleta (localidade, município, estado e coordenadas geográficas), o nome do coletor, e as características físicas do ambiente (tipo do solo). Já em relação às características da planta e da coleta propriamente dita, anotou-se: o hábito da planta, flores e frutos e outras características marcantes que auxiliarão na hora da identificação.

\section{Preparação do material}

Essa etapa consiste basicamente no processo de herborização dos espécimes vegetais coletados, sendo processados da seguinte forma: após a coleta, os espécimes vegetais foram prensados entre folhas de jornal e papelão, posteriormente secos a temperatura ambiente (Fig. 3-B). Com o material desidratado, os mesmos foram fixados em cartolina, de tamanho apropriado ( $28 \times 42$ $\mathrm{cm}$ ), onde receberam etiquetas com dados informativos a respeito do espécime, constituindo desta forma a exsicata. 


\section{Identificação do material botânico}

Nesta etapa, os espécimes coletados foram identificados em família, gênero e espécie por meio de comparação com o material tipo, bibliografias especializadas, artigos científicos, livros, herbários virtuais e chaves taxonômicas (Fig. 3-C). O estado de conservação das espécies foi avaliado, de acordo com os critérios da União Internacional para Conservação da Natureza (IUCN), que tem como base a lista de espécies, nas informações obtidas nas etiquetas dos herbários e das observações de ocorrência durantes as expedições.

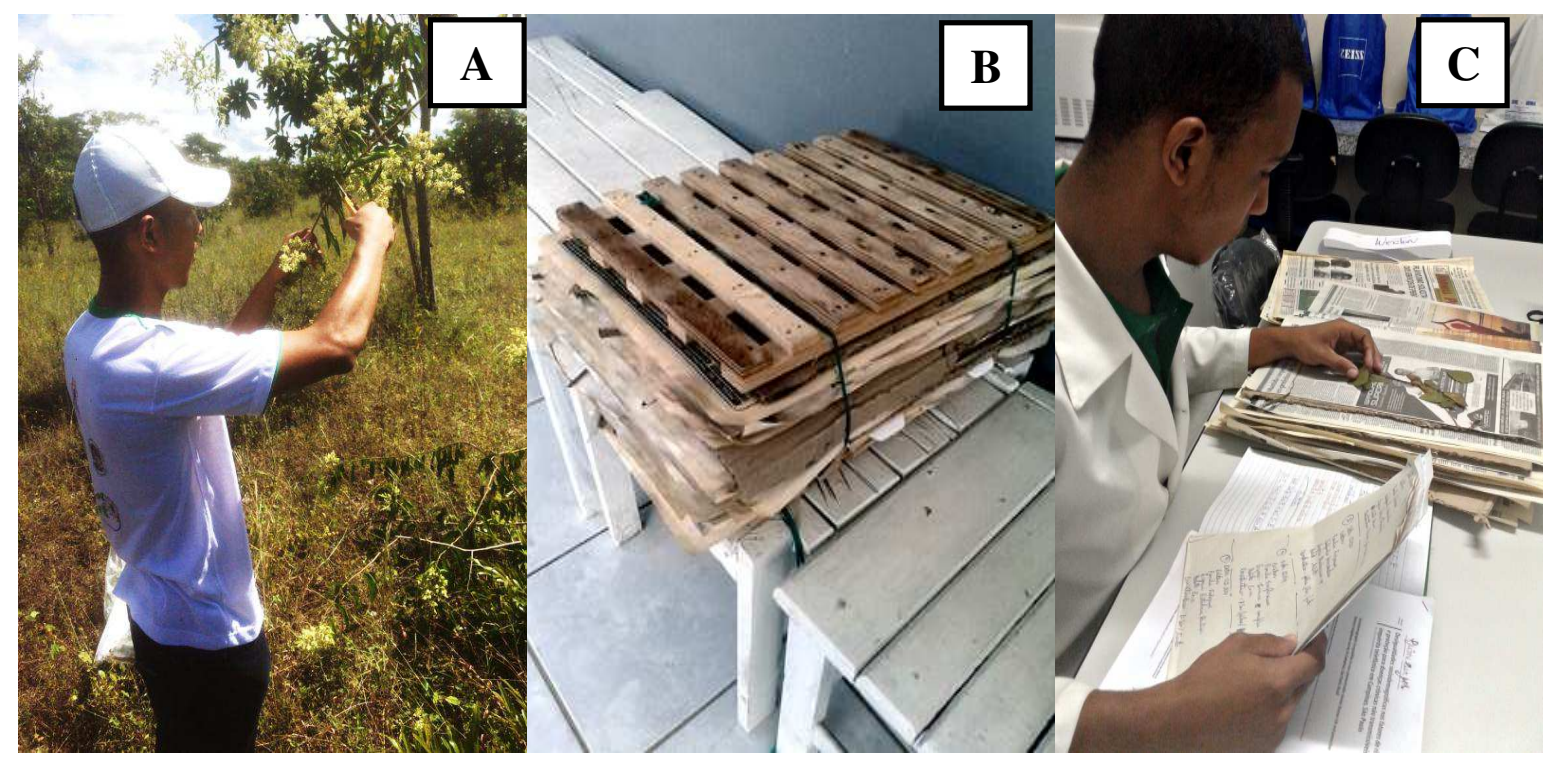

FIGURA 3. Etapas que compõem o método florístico. A) coleta dos espécimes vegetais utilizando a tesoura de poda. B) Herborização do material coletado. C) Identificação dos espécimes coletados em campo. Fonte: CAMELO-JÚNIOR (2016).

\section{RESULTADOS E DISCUSSÃO}

Com o levantamento realizado foram obtidas 89 espécies, distribuídos em 78 gêneros e 32 famílias botânicas (Quadro 1). Os dados amostram uma grande variedade vegetacional, sendo 31 famílias de Angiospermas e apenas uma família de Monilófitas (Thelypteridaceae). Os estudos sobre a estrutura das formações florestais são de fundamental importância, pois oferecem subsídios para a compreensão da estrutura e da dinâmica destas formações, parâmetros imprescindíveis para o manejo e regeneração das diferentes comunidades vegetais (CHAVES et al., 2013).

QUADRO 1. Espécies amostradas na Área de Proteção Ambiental Municipal do Buriti do Meio, Caxias-Maranhão.

\begin{tabular}{|c|c|}
\hline FAMILIA & ESPÉCIE \\
\hline ACANTHACEAE & Justicia sp. \\
\cline { 2 - 2 } & Justicia aequilabris (Nees) Lindau. \\
\hline AMARYLLIDACEAE & Zephyranthes sp. \\
\hline ANACARDIACEAE & Anacardium occidentale L. \\
\cline { 2 - 2 } & Astronium graveolens Jacq. \\
\hline
\end{tabular}




\begin{tabular}{|c|c|}
\hline \multirow{3}{*}{ ANNONACEAE } & Annona crassiflora Mart. \\
\hline & Unonopsis guatterioides (A.DC.) R.E.Fr. \\
\hline & Xylopia aromática (Lam.) Mart. \\
\hline \multirow{3}{*}{ APOCYNACEAE } & Himatanthus drasticus (Mart.) Plumel \\
\hline & Himatanthus obovatus (Müll. Arg.) Woodson \\
\hline & Plumeria rubra L. \\
\hline \multirow{3}{*}{ ARECACEAE } & Attalea phalerata L.f. \\
\hline & Bactris simplicifrons Mart. \\
\hline & Mauritia flexuosa Mart. ex Spreng. \\
\hline \multirow{4}{*}{ ASTERACEAE } & Ageratum conyzoides L. \\
\hline & Elephantopus mollis Kunth. \\
\hline & Rolandra fruticosa (L.) Kuntze \\
\hline & Tilesia baccata (L.f.) Pruski \\
\hline CACTACEAE & Cereus jamacaru DC. \\
\hline CARYOCARACEAE & Caryocar brasiliense Cambess. \\
\hline CLUSIACEAE & Platonia insignis Mart. \\
\hline CYPERACEAE & Killinga brevifolia Rottb. \\
\hline \multirow{4}{*}{ COMBRETACEAE } & Combretum leprosum Mart. \\
\hline & Terminalia argentea Mart. \\
\hline & Terminalia catapa L. \\
\hline & Terminalia fagifolia Mart. \\
\hline COMMELINACEAE & Commelina erecta $\mathrm{L}$. \\
\hline \multirow{3}{*}{ CONVOLVULACEAE } & Evolvulus alsinoides $\mathrm{L}$. \\
\hline & Ipomoea alba L. \\
\hline & Ipomoea quamoclit L. \\
\hline DILLENIACEAE & Curatella americana $\mathrm{L}$. \\
\hline EBENACEAE & Diospyros hispida A. DC. \\
\hline EUPHORBIACEAE & Manihot esculenta Crantz. \\
\hline \multirow{19}{*}{ FABACEAE } & Aeschynomene histrix Poir. \\
\hline & Aeschynomene indica L. \\
\hline & Andira vermifuga (Mart.) Benth. \\
\hline & Bauhinia dubia G. Don \\
\hline & Libidibia ferrea (Mart.) L.P.Queiroz. \\
\hline & Chamaecrista viscosa (Kunth) H. S. Irwin \& Barneby \\
\hline & Crotalaria retusa $\mathrm{L}$. \\
\hline & Delonix regia (Bojer ex Hook.) Raf. \\
\hline & Desmodium barbatum (L.) Benth. \\
\hline & Dimorphandra gardneriana Tul. \\
\hline & Galactia jussianeae Kunth. \\
\hline & Hymenaea stigonocarpa Mart. Ex Hayne \\
\hline & Inga laurina (Sw.) Willd. \\
\hline & Indigofera suffruticosa Mill. \\
\hline & Leptolobium dasycarpum Vogel. \\
\hline & Periandra heterophylla Benth. \\
\hline & Mimosa sensitiva L. \\
\hline & Parkia platycephala Benth. \\
\hline & Plathymenia reticulata Benth. \\
\hline
\end{tabular}




\begin{tabular}{|c|c|}
\hline & Pterodon emarginatus Vogel. \\
\hline & Senna obtusifolia (L.) H. S. Irwin \& Barneby \\
\hline & Senegalia polyphylla (DC.) Britton \& Rose \\
\hline & Stryphnodendron coriaceum Benth. \\
\hline & Stylosanthes angustifólia Vogel. \\
\hline & Stylosanthes capitata Vogel. \\
\hline & Tachigali vulgaris L. G. Silva \& H. C. Lima. \\
\hline & Tachigali glauca Tul. \\
\hline & Tephrosia purpurea (L.) Pers. \\
\hline & Vatairea macrocarpa (Benth.) Ducke \\
\hline LAURACEAE & Endlicheria sp. \\
\hline \multirow{7}{*}{ MALVACEAE } & Abutilion purpurascens K. Schum. \\
\hline & Calyptranthes pulchella A. St-Hil. \\
\hline & Pavonia cancellata (L.) Cav. \\
\hline & $\begin{array}{l}\text { Pseudobombax marginatum (A. St.-Hil., Juss. \& } \\
\text { Cambess.) A. Robyns }\end{array}$ \\
\hline & Solanum lycocarpum DC. \\
\hline & Sterculia sp. \\
\hline & Sterculia striata A. St.-Hil. \& Naudin \\
\hline \multirow[t]{2}{*}{ MELASTOMATACEAE } & Miconia albicans (Sw.) Triana \\
\hline & Tibouchina aspera Aubl. \\
\hline MORACEAE & Ficus sp. \\
\hline \multirow{4}{*}{ MYRTACEAE } & Eugenia uniflora L. \\
\hline & Eugenia flavescens DC. \\
\hline & Myrcia sp. \\
\hline & Myrcia tomentosa (Aubl.) DC. \\
\hline \multirow{2}{*}{ OCHNACEAE } & Ouratea sp. \\
\hline & Ouratea hexasperma (A.St.-Hil.) Baill. \\
\hline ORCHIDACEAE & Catasetum barbatum (Lindl.) Lindl. \\
\hline \multirow[t]{2}{*}{ TURNERACEAE } & Piriqueta duarteana (Cambess.) Urb. \\
\hline & Turnera umifolia $\mathrm{L}$. \\
\hline RUBIACEAE & Cordiera sessilis (Vell.) Kuntze \\
\hline FLACOURTIACEAE & Casearia sylvestris Sw. \\
\hline SOLANACEAE & Solanum grandiflorum Ruiz \& Pav. \\
\hline \multirow[t]{2}{*}{ VOLCHYSIACEAE } & Qualea parviflora Mart. \\
\hline & Salvertia convallariodora A. St.-Hil. \\
\hline XYRIDACEAE & Xyris macrocephala Vahl. \\
\hline THELYPTERIDACEAE & Thelypteris interrupta (Willd.) K. Iwats. \\
\hline
\end{tabular}

Tratando-se da representatividade quanto aos dados florísticos, as Famílias mais representativas foram Fabaceae com 29 espécies, Malvaceae com sete espécies, seguida por Myrtaceae, Asteraceae e Combretaceae com quatro espécies cada (FIGURA 4). Considerando os resultados de representatividade das famílias neste levantamento, corroboram com os estudos florísticos, que em geral, as famílias Fabaceae e Myrtaceae são predominantes nos inventários florístico do neotrópico (GENTRY, 1988; 1990). Malvaceae possui distribuição predominantemente entre as regiões dos trópicos, especialmente na América do Sul, 
com alguns representantes em regiões temperadas e inclui cerca de 250 gêneros e 4200 espécies, ocorrendo no Brasil cerca de 80 gêneros e 400 espécies (SOUZA \& LORENZI, 2008).

O cerrado sensu lato já pode ser, portanto, considerado um ambiente tropical que detêm elevada parcela de conhecimento sobre sua vegetação e chama atenção aos possíveis processos responsáveis pelos padrões ecológicos até agora identificados servindo de leito para uma considerável diversidade da família Fabaceae (NETTESHEIM et al., 2010). Os dados do trabalho corroboram com os trabalhos de SOUSA-FILHO (2006) e NERES \& CONCEIÇÃO (2010).

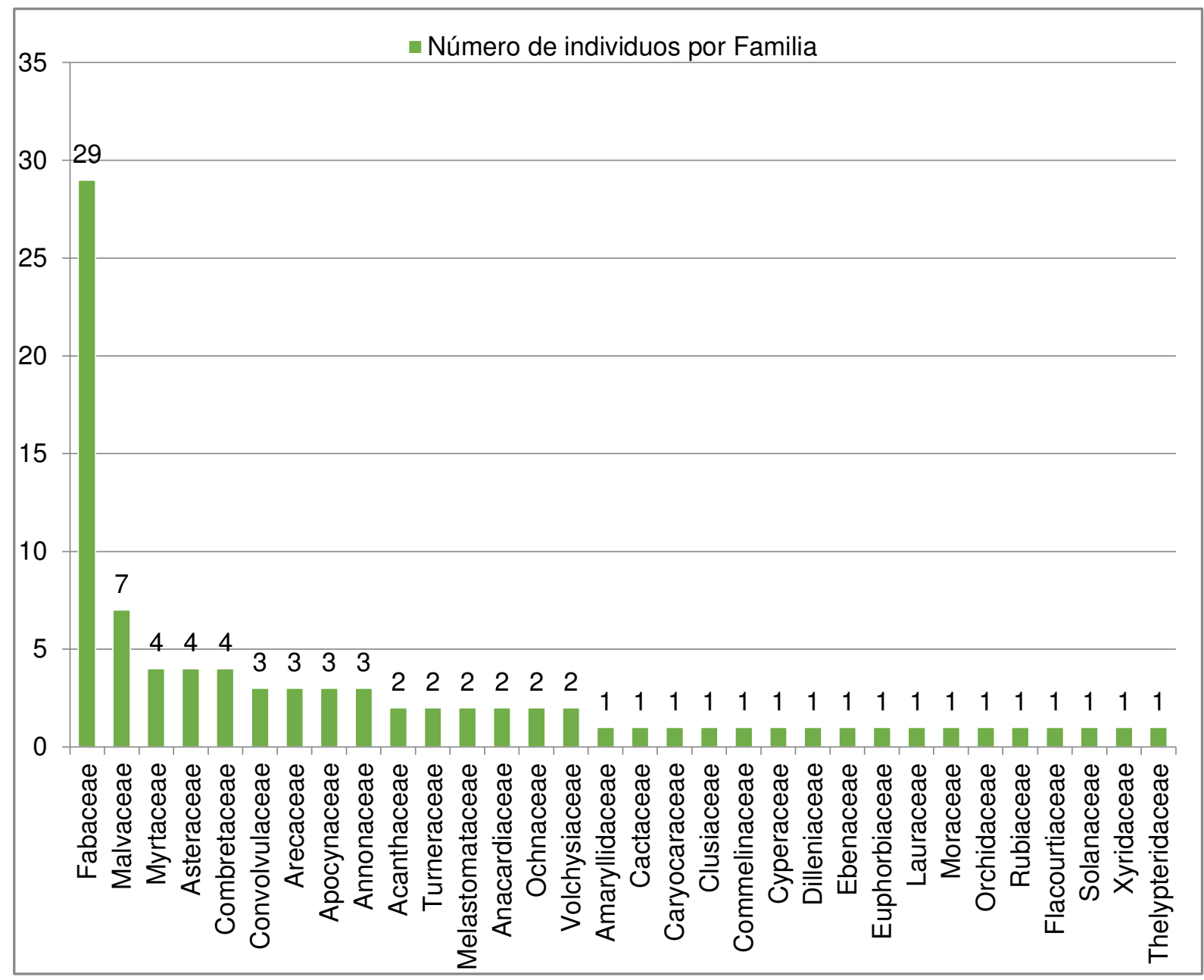

FIGURA 4. Representação gráfica da Distribuição de Famílias encontradas no Povoado Buriti do Meio. Fonte: autores (2017)

Com o levantamento florístico elencou-se uma nova ocorrência para o Estado do Maranhão, espécie esta pertencente à família Fabaceae, Aeschynomene indica. Para Aeschynomene estima-se cerca de 350 espécies, distribuídas nos trópicos e em locais relativamente mais quentes das zonas temperadas sendo que, para o continente americano, foram listadas 160 espécies (KLITGAARD \& LAVIN, 2005). Para o Brasil ocorrem 49 espécies, o dado aumenta para 13 espécies do gênero para o Maranhão, segundo a FLORA DO BRASIL 2020 (2017).

Tratando-se de gêneros, Aeschynomene, Justicia, Sterculia, Stylosanthes, Ipomoea. Hymathantus, Terminalia, Tachigali, Ouratea, Eugenia e Myrcia foram os AGRARIAN ACADEMY, Centro Científico Conhecer - Goiânia, v.4, n.7; p. 2752017 
gêneros mais representativos ambos com duas espécies cada, as demais espécies amostraram apenas um epíteto. Entre os gêneros de leguminosas arbóreas neotropicais, Tachigali destaca-se pela elevada riqueza de espécies e pela abundância em ambientes de margens de rios e em formações florestais em regeneração (SILVA \& LIMA, 2007). A família Combretaceae compreende 18 gêneros, sendo Combretum e Terminalia os mais abundantes, com cerca de 370 e 200 espécies, respectivamente, as quais são largamente distribuídas no Oeste e Sul da África e amplamente utilizadas na medicina popular (MASOKO, 2007). Algumas das espécies do gênero Justicia também possuem importância forrageira e ecológica, sendo abundantes e bem distribuídos em florestas úmidas e dominantes em ambientes semiáridos (EZCURRA, 2002).

O gênero Myrcia apresenta 374 espécies (GOVAERTS et al., 2008), ocorre desde a América Central até o norte da Argentina (LANDRUM \& KAWASAKI, 1997). No Brasil são encontradas 214 espécies (LEGRAND, 1968). Indivíduos pertencentes ao gênero Aeschynomene são citadas como fixadoras de nitrogênio, apícolas, tóxicas para suínos, ornamentais, forrageiras e para a fabricação de boias de flutuação (RUDD, 1955; TIMM \& RIET-CORREA, 1997; POTT et al., 2000; ULIBARRI et al., 2002; ALVES, 2008). O gênero Ipomoea possui entre 600-700 espécies com distribuição cosmopolita e é o gênero com maior riqueza especifica pertencente à família Convolvulaceae (AUSTIN \& CAVALCANTI, 1982).

Quanto à espécimes a espécie mais representativa foi Pseudobombax marginatum com 20 espécimes elencadas, Parkia platycephala com 18 espécimes elencadas, seguido por Eugenia uniflora com 10 espécimes, Hymathantus drasticus com 5 espécimes e Pterodon emarginatus, Caryocar brasiliensis e Casearia sylvestris com 4 espécimes cada. A utilidade das Pseudobombax está, em geral, relacionada às suas sementes $e$, em grande parte, à paina originada da parede interna do endocarpo dos seus frutos, da qual deve ter-se originado o nome vernacular "paineira", como são chamadas em diversas regiões do Brasil (BRAGA, 1960; LORENZI, 2002). $P$. emarginatus e $P$. platycephala são árvores típicas do cerrado, onde são características desse domínio fitogeográfico, sendo que $P$. platycephala também conhecida como faveira, é uma planta perenifólica, com ocorrência na região amazônica, sul da Bahia e norte do Espírito Santo, chegando a uma altura de até 30 metros e troncos que podem chegar a $100 \mathrm{~cm}$ de diâmetro (LORENZI, 2002).

\section{CONCLUSÃO}

Pode-se inferir que a amostra de espécies listadas para a área de estudo, foi expressivo, com destaque para família Fabaceae, por apresentar elementos florísticos distribuídos por toda a APA de pesquisa, contribuindo com 29 espécies.

Aeschynomene indica é uma nova ocorrência para o estado do Maranhão, o que vem reforçar a importância dos levantamentos florísticos, que contribuem para a ampliação do entendimento da vegetação e reconhecimento de espécies que até então não haviam sido registradas.

Por fim, demonstra-se através dos dados que a área estudada é bastante diversa, com predominância do hábito de crescimento do tipo árvore, por se tratar de um fragmento com fisionomia do tipo cerradão. A área está sujeita à uma intensa pressão antrópica que consequentemente acarreta drásticas mudanças em sua composição florística. Medidas de manejo e conservação, bem como, o desenvolvimento de estratégias que possibilitem a integridade da Área de Proteção AGRARIAN ACADEMY, Centro Científico Conhecer - Goiânia, v.4, n.7; p.276 2017 
Ambiental do Buriti do Meio, devem ser tomadas pelo poder público a fim de preservar o patrimônio vegetal presente.

\section{REFERÊNCIAS}

ALVES, E. M. Identificação da flora e caracterização do mel orgânico de abelhas africanizadas das ilhas Floresta e Laranjeira, do alto rio Paraná. Tese (Doutorado em Zootecnia) - Centro de Ciências Agrárias. Universidade Estadual de Maringá, Maringá, p. 58, 2008.

AUSTIN, D. F.; CAVALCANTE, P. B. Convolvuláceas da Amazônia. Publicações Avulsas do Museu Paraense Emilio Goeldi. v. 36, p. 134, 1982.

BRAGA, R. Plantas do Nordeste, especialmente do Ceará. Ed. Universitária UFRN. $4^{\circ}$ ed. Natal, p. 540, 1960.

CHAVES, A. C. D. G.; SANTOS, R. M. S.; SANTOS, J. O.; FERNANDES, A. A.; MARACAJÁ, P. B. A importância dos levantamentos florístico e fitossociológico para a conservação e preservação das florestas. ACSA - Agropecuária Científica no Semiárido, v. 9, n. 2, p. 43-48, 2013.

CONCEIÇÃO, G. M.; CASTRO, A. A. J. F. Fitossociologia de uma área de cerrado marginal, Parque Estadual do Mirador, Mirador, Maranhão. Scientia Plena. v. 5, $n^{\circ}$ 10, p. 16, 2009.

EZCURRA, C. El género Justicia (Acanthaceae) en Sudamérica Austral. Annals of the Missouri Botanical Garden. n 89, p. 225-280, 2002.

FELFILI, J. M.; EISENLOHR, P. V.; MELO, M. M. R. F.; ANDRADE, L. A.; NETO, J. A. A. M. Fitossociologia no Brasil: Métodos e Estudos de Casos. Editora UFV. Viçosa, MG. p. 174-212, 2013.

FELFILI, J. M.; SILVA JÚNIOR, M. C. Biogeografia do Bioma Cerrado: estudo fitofisionômico da Chapada do Espigão Mestre do São Francisco. Universidade de Brasília, Faculdade de Tecnologia, Departamento de Engenharia Florestal, Brasília. p. 35-36, 2001.

FERNANDES, A. Conexões florísticas do Brasil. Fortaleza: Banco do Nordeste. p. 134, 2003.

FLORA DO BRASIL 2020. Jardim Botânico do Rio de Janeiro. 2017. Disponível em: < http://floradobrasil.jbrj.gov.br/ >. Acesso em: 06/07/2017.

GENTRY, A. H. Changes in plant community diversity and floristic composition on environmental and geographical gradients. Annals of the Missouri Botanical Garden, n.75, p.1-34, 1988.

GENTRY, A. H. Floristic similarities and differences between Southern Central America and upper and Central Amazonia. In: GENTRY, A. H. Four neotropical rain forests. Yale University Press, New Haven, p.141-160, 1990.

AGRARIAN ACADEMY, Centro Científico Conhecer - Goiânia, v.4, n.7; p. 2772017 
GOMES, B. Z.; MARTINS, F. R.; TAMASHIRO, J. Y. Estrutura do cerradão e da transição entre cerradão e floresta paludícola num fragmento da International Paper do Brasil Ltda., em Brotas, SP. Revista Brasil. Bot. v. 27, n. 2, p. 249-262, 2004.

GOVAERTS, R.; SOBRAL, M.; ASHTON, P.; BARRIE, F.; HOLST, B. K.; LANDRUM, L. R.; MATSUMOTO, K.; MAZINE, F. F.; NIC LUGHADHA, E.; PROENÇA, C.; SOARES-SILVA, L. H.; WILSON, P. G.; LUCAS, E. World checklist of Myrtaceae. Royal Botanic Gardens, Kew. p. 455, 2008.

IBGE - Instituto Brasileiro de Geografia e Estatística. Censo: 2010. Disponível em: http:<//www1.ibge.gov.br/cidadesat/topwindow.htm?1>. Acesso em: 10/02/2016.

KLITGAARD, B. B.; LAVIN M. Tribe Dalbergieae sens. lat. In: LEWIS, G.P.; SCHRIRE, B.; MACKINDER, B.; LOCK, M. Legumes of the world. Royal Botanic Gardens, Kew. Pp. 307-335, 2005.

LANDRUM, L. R.; KAWASAKI, M. L. The genera of Myrtaceae in Brazil: an illustrated synoptic treatment and identification keys. Brittonia, v. 49, n. 4, p. 508-536, 1997.

LEGRAND, C. D. Las Mirtaceas del Uruguay III. Boletín de Facultad de Agronomía de Montevideo, n. 101, p. 1-80, 1968.

LORENZI, H. Árvores Brasileiras: Manual de Identificação e Cultivo de Plantas Arbóreas Nativas do Brasil. 4 Ed. Plantarum. São Paulo. v. 2, p. 384, 2002.

MASOKO, P.; PICARD, J.; ELOFF, J. N. S. Afr. J. Bot., v. 73, p. 173, 2007.

MONTES, M. L.; Zoneamento Geoambiental do Estado Do Maranhão - Diretrizes Gerais Para a Ordenação Territorial. Ministério de Planejamento, Orçamento e Coordenação. Fundação Instituto Brasileiro de Geografia e Estatística. Salvador. 1997. 44p.

NERES, L. P.; CONCEIÇÃO, G. M. Florística e Fitossociologia da Área de Proteção Ambiental Municipal do Inhamum, Caxias, Maranhão, Brasil. Cadernos de Geociências, v. 7, n. 2, p. 122-130, 2010.

NETTESHEIM, F. C.; CARVALHO, D. C.; FONSECA, C. C.; NUNES, R. S.; CAVALCANTI, D. M.; GABRIEL, M. M.; MENESES, L. F. T. Estrutura e Florística do Estrato arbóreo no Cerrado sensu stricto de Buritis, Minas Gerais, Brasil. Revista Rodriguésia. UFRJ. RJ. v. 61, n.4, p. 731-774, 2010.

POTT, A.; POTT, V. J.; SOUZA, T. W. Plantas aquáticas do Pantanal. Campo Grande: Embrapa Gado de Corte. p. 404, 2000.

RUDD, V. E. The american species of Aeschynomene. Bulletin of the United States National Herbarium. n. 32, p. 1-172, 1955.

SALIS, S. M.; TAMASHIRO, J. Y.; JOLY, C. A. Florística e fitossociologia do estrato AGRARIAN ACADEMY, Centro Científico Conhecer - Goiânia, v.4, n.7; p. 2782017 
arbóreo de um remanescente de mata ciliar do rio Jacaré-Pepira, Brotas, SP. Revista Brasileira de Botânica, v. 17, p. 93-103, 1994.

SANTOS, L. J.; MARMONTEL, C. V. F.; MARTINS, T. M.; MELO, A. G. C. Fitossociologia de Cerrado Sensu Stricto localizado no Município de Carbonita-MG. R. C. E. E. F., v.15, n.1, p. 77-90, 2010.

SILVA FILHO, J. F. Florística e Fitossociologia da Área de Proteção Ambiental Municipal do Inhamum do Município de Caxias e Comparação com outras Áreas do Estado do Maranhão, Brasil. Monografia (Graduação em Biologia) Universidade Estadual do Maranhão, Centro de Estudos Superiores de Caxias, p. 68, 2006.

SILVA, L. F. G.; LIMA, H. C. Mudanças nomenclaturais no gênero Tachigali Aubl. (Leguminosae-Caesalpinioideae) no Brasil. Rodriguésia, Rio de Janeiro, v.58, n.2, p. 397-401, 2007.

SILVA, W. F. N. Mapa Hidrográfico e Localização da área de estudo, pontos de coletas, Buriti do Meio, Caxias/MA. Google Earth Pro. ArcGis 9.3. Consultado em: 22/01/2016. Werton Francisco Nobre Silva. 2016.

SOUZA, V. C.; LORENZI, H. Botânica Sistemática: Guia ilustrado para identificação das famílias de Fanerógamas nativas e exóticas no Brasil, baseado em APG II. 2. ed. Nova Odessa: Instituto Plantarum de Estudos da Flora, p. $704,2008$.

TIMM, C. D.; RIET-CORREA, F. Plantas tóxicas para suínos. Ciência Rural. v. 27, n. 3, p. 521-528, 1997.

ULIBARRI, E. A.; SOSA, E. V. G.; CIALDELLA, A. M.; FORTUNATO, R. H.; BAZZANO, D. Leguminosas: nativas e exóticas. In: HURREL, J. A.; LAHITTE, H. B. Biota riplatense VII. Buenos Aires: L.O.L.A. p. 320, 2002. 